\title{
ESTUDO COMPARATIVO ENTRE ANTIMONIATO DE MEGLUMINA, ISOTIANATO DE PENTAMIDINA E SULFATO DE AMINOSIDINE, NO TRATAMENTO DE LESÕES CUTÂNEAS PRIMÁRIAS CAUSADAS POR LEISHMANIA (VIANNIA) BRAZILIENSIS
}

\author{
Dalmo Correia, Vanize O. Macêdo, Edgar M. Carvalho, Aldina Barral, \\ Albino V. Magalhães, Maria Virgínia A. de Abreu, \\ Maria de La Glória Orge Orge e Philip Marsden
}

\begin{abstract}
Com o objetino de comparar a eficácia, a tolerabilidade e a toxicidade do antimoniato de meglumina, do sulfato de aminosidine e do isotianato de pentamidine no tratamento de lesôes cutâneas primárias causadas por Leishmania (Viannia) braziliensis foi realizado um estudo de campo, aberto, randomizado, na área endêmica de Corte de Pedra-Babia. De outubro de 1992 a janeiro de 1993, foram tratados 46 pacientes, distribuídos em três grupos dois de $15 \mathrm{e} u \mathrm{~m}$ de 16 pacientes. Todos os pacientes realizaram exames clinnico, parasitológico, histopatológico e imunológico, como critério diagnóstico. Todos os pacientes foram tratados pela via intramuscular. O Grupo 1 recebeu pentamidina na dose de $4 \mathrm{mg} / \mathrm{kg} / \mathrm{dia}$, em dias alternados, no total de 8 aplicações; o Grupo 2 aminosidine na dose de $20 \mathrm{mg} / \mathrm{kg} /$ dia, por 20 dias; o Grupo 3 , meglumina na dose de 10mg/kg/dia, por 20 dias. Definiu-se como falha terapêutica a permanência de lesões ulceradas, após quatro meses de tratamento. Ocorreram cinco casos de falba terapêutica assin distribuidos: dois casos no Grupo 1, um caso no Grupo 2 e dois no Grupo 3, ao final do primeiro ano de seguimento. Na avaliação após 3 anos foram relistos 15 pacientes, $5 \mathrm{em}$ cada Grupo; exceto um do Grupo 3, todos continuaram curados. Não bouve diferença estatistica entre os resultados dos três esquemas utilizados.
\end{abstract}

Palanas-chaves: Tratamento da leishmaniose tegumentar americana. Antimonial pentalalente. Pentamidina. Aminosidine.

A leishmaniose tegumentar situa-se entre as grandes endemias existentes no Brasil e na América Latina ${ }^{11}{ }^{20}$. Apesar das falhas na notificação compulsória, é crescente o número de casos novos da doença em nosso país. No que se refere à distribuição geográfica mundial, a leishmaniose é prevalente nos quatro continentes, sendo considerada endêmica em

Núcleo de Medicina Tropical e Nutrição da Universidade de Brasília, Brasília, DF. Serviço de Imunologia, Hospital Universitário Professor Edgar Santos, Universidade Federal da Bahia, Salvador, BA.

Suporte financeiro: Núcleo de Medicina Tropical e Nutrição. Convênio FUB/OMS-28100121; NIH Grant: A1-30639 e PCDEN/Ministério da Saúde do Brasil.

Endereço para correspondência: Prof. Dalmo Correia Filho. Disciplina de Doenças Infecciosas e Parasitárias/FM'T'M, Caixa Postal 118,38001-970 Uberaba,MG.Tel.:(034) 312-7722 r: 1285; Fax: (034) 312-7722 r: 1279.

Recebido para publicação em 02/04/96.
82 países. Anualmente, a nível mundial, o número de casos novos de leishmaniose é estimado em $600 \mathrm{mil}$, com uma prevalência de 12 milhôes de pessoas, uma população de risco avaliada entre 350 a 400 milhões, e uma taxa de mortalidade em torno de $1000^{11}$.

No Brasil, o número de casos novos de leishmaniose tegumentar americana (LTA) notificados anualmente, no período entre 1980 e 1993, mostra uma ascensão constante, com um total de 4.408 em 1980, contra $26.611 \mathrm{em}$ 1987. A partir de 1987, há um decréscimo nítido nesse último número, tendo sido notificados até o mês de agosto de 1993, um total de 10.602 casos novos.

A $L(V) b$ é o agente etiológico mais importante de LTA no Novo Mundo, devido à freqüência com que ocasiona comprometimento de mucosas. Clinicamente, se faz com relativa facilidade o diagnóstico de LTA, devido às suas características, tais como: lesão ulcerada, com 
Comreia D, Macêdo VO, Canialbo EM, Barral A, Magalbães AV, Abreu MVA, Orge MLGO, Marsden P. Estudo comparativo entre antimoniato de meglumina, isotianato de pentamidina e sulfato de aminosidine, no tratamento de lesöes cutâneas causadas por Leishmania (Viannia) braziliensis. Revista da Sociedade Brasileira de Medicina Tropical 29:447-453, set-out, 1996.

bordas elevadas e grau de necrose variávelit. Contudo, o isolamento do agente etiológico se faz com dificuldade.

Cuba e cols $s^{9}$ e Rosa e $\operatorname{cols}^{26}$ demonstraram que o agente etiológico predominante na área endêmica de Três Braços, BA, é a Leisbmania (Viannia) braziliensis $L(V) b$. No que se refere ao vetor, nessas áreas endêmicas, é provável que seja a Lutzomia whitmani $i^{28}$.

A LTA se apresenta sob forma clínica variável, com características evolutivas peculiares a cada paciente. Mais de 90\% dos casos correspondem à forma cutânea da doença e em menos de $3 \%$ ao comprometimento de mucosas $^{18}$. O envolvimento de mucosas, no processo de evolução natural da doença, é extremamente variável e de difícil tratamento ${ }^{i 5}$.

A leishmaniose causada por $L(V) b$ é de difícil controle clínico e no que se refere às alteraçôes patológicas, admite-se que ela induz a um grau de necrose mais intenso, passando por fases distintas de um mesmo processo evolutivo, quando comparado a alteraçoes histopatológicas causadas por outras espécies ${ }^{13}$.

Recentemente, tem-se evidências clínicas e laboratoriais da ocorrência precoce de disseminação linfática de leishmânias para linfonodos satélites, reforçando a necessidade de uma abordagem terapêutica precoce ${ }^{22}$. Atualmente, aceita-se que a ocorrência de leishmaniose clinicamente manifesta esteja na dependência de fatores inerentes ao parasita, da resistência natural do hospedeiro e da magnitude de sua resposta imunológica ${ }^{3}$. Assim sendo, a doença passa a ter um amplo espectro de variação clínica, com prognóstico variável, indo desde formas que evoluem para cura espontânea, até às formas resistentes à terapêutica convencional ${ }^{58}$.

Para a terapêutica específica da LTA, ainda não se dispõe de um composto farmacológico que seja igualmente efetivo, ou superior ao antimonial pentavalente. Introduzido no arsenal terapêutico por Gaspar Vianna em 1912 , sob forma de composto trivalente, o antimonial permanece ainda como droga de primeira escolha, apesar da administraçào por via parenteral, do difícil manuseio, da sua potencial toxicidade e do mecanismo de ação ainda não totalmente esclarecido. Parece ser consenso que seu mecanismo de ação se faça graças a uma possível inibição de enzimas glicolíticas, ocorrendo, assim, a beta oxidação de ácidos graxos ${ }^{21}$. Tradicionalmente, os compostos farmacológicos utilizados como segunda escolha para o tratamento da LTA são: anfotericina $B$ e pentamidina, sendo a sua indicação restrita aos casos de falha terapêutica ao antimonial pentavalente, devido à dificuldade no seu manuseio e à toxicidade ${ }^{29}$.

Recentemente, no Quênia, tem-se empregado o sulfato de aminosidine no tratamento da leishmaniose visceral, isolado ou em associação com o estibogluconato de sódio?. Também se tem demonstrado que a paromomicina é ativa contra leishmaniose cutânea causada por $L$. brasiliensis e L. mexicana ${ }^{25}$.

\section{MATERIAL E MÉTODOS}

Realizou-se um estudo prospectivo, aberto, em face à toxicidade dos compostos utilizados e randomizado. O protocolo foi aprovado pela comissão de ética da Universidade de Brasília e Universidade Federal da Bahia e foi obtido consentimento, por escrito, dos pacientes e/ou seus responsáveis.

Entre outubro de 1992 e janeiro de 1993, procedeu-se ao atendimento dos pacientes, no posto de saúde de Corte de Pedra, Bahia, área endêmica para leishmaniose tegumentar americana (LTA) e simultaneamente 46 pacientes foram randomicamente alocados em três grupos, 2 de 15 e um de 16 , desde que preenchessem os critérios de inclusão: lesões cutâneas primárias compativeis com LTA, idade entre 12 e 60 anos, númeto máximo de cinco úlceras e tempo de evolução menor de seis meses.

Todos os pacientes foram submetidos a exames clínico e otorrinolaringológico detalhados, realização de eletrocardiograma, urina tipo I, (elementos anormais e sedimento) e coleta de sangue para obtenção de soro, com vistas à avaliação bioquímica de uréia, creatinina, fosfatase alcalina, transaminase glutâmicooxalacética e pirúvica, glicemia e reação de imunofluorescência indireta, segundo técnica padronizada no Laboratório de Imunologia da Universidade Federal da Bahia ${ }^{31}$, pré e póstratamento e, a cada três meses, por um ano. Em todos os pacientes, foi feita intradermorreação de Montenegro (IDRM) ${ }^{1023}$, biópsia das lesões e cultura do material aspirado das lesões e processadas no Laboratório de Patologia de Universidade de Brasília/UnB e Laboratório de Imunologia da Universidade Federal da 
Correia D, Macêdo VO, Carvalho EM, Barral A, Magaibães AV, Abreu MVA, Orge MLGO, Marsden P. Estudo comparativo entre antimoniato de meglumina, isotianato de pentamidina e sulfato de aminosidine, no tratamento de lesōes cutâneas causadas por Leishmania (Viannia) braziliensis. Revista da Sociedade Brasileira de Medicina Tropical 29:447-453, set-out, 1996.

Bahia/UFBA, respectivamente, segundo técnicas padronizadas ${ }^{13}{ }^{18}$. A caracterização dos isolados foi feita no Laboratório de Imunologia da UFBA, utilizando-se anticorpos monoclonais e isoenzimas ${ }^{122-1}$.

Procedeu-se à administração dos esquemas terapêuticos a nível ambulatorial, diário e supervisionado nos seguintes esquemas: Grupo 1 recebeu pentamidina na dose de $4 \mathrm{mg} / \mathrm{kg} / \mathrm{dia}, \mathrm{IM}$, em locais variados, em dias alternados, no total de 8 aplicações ${ }^{22}$; o Grupo 2 recebeu aminosidine na dose de $20 \mathrm{mg} / \mathrm{kg} / \mathrm{dia}$, IM, em locais variados, durante $20 \mathrm{dias}^{6}$ e o Grupo 3, meglumina na dose de $10 \mathrm{mg} / \mathrm{kg} / \mathrm{dia}$, IM, em diferentes locais, por 20 dias $^{16}$.

A análise estatística foi feita utilizando-se um banco de dados, a partir das fichas epidemiológicas, no programa EPIINFO, versāo 5.0 (CDC Atlanta e WHO, Geneva). Todos os dados de distribuição normal nos três grupos de pacientes, bem como aqueles agrupados por intervalos e o tempo decorrido até à cicatrização total, foram analisados por análise de variância (ANOVA-SAS Institute Inc, 1985). Os exames laboratoriais, com o Teste $\mathrm{T}$ de Student nos três grupos terapêuticos. Todos os resultados tiveram por referência um nível de significância de $\mathrm{p} \backslash<0,05$.

\section{RESULTADOS}

As características gerais da população estudada nos três grupos estão na Tabela 1 . A idade variou de 12 a 56 anos, com predominância do sexo masculino $(65 \%)$ e a ocupação principal era o trabalho na lavoura $(72 \%)$. As lesões predominaram nos membros inferiores (72\%), sendo que $42 \%$ tiveram duração menor ou igual a 30 dias e $78 \%$ dos pacientes apresentaram apenas uma lesão.

A Tabela 2 mostra a distribuição dos procedimentos diagnósticos nos três grupos, realizados pré-tratamento, onde se observa que em $82 \%$ dos pacientes a IDRM foi positiva; em $78 \%$ dos fragmentos de biópsia foi demonstrada a presença de amastigotas ao estudo histopatológico. Apenas 19\% das culturas dos aspirados das lesões foram positivas para leishmânia, enquanto na impressão em lâmina pôde-se detectar a presença de amastigotas em 56\% dos pacientes. A reação de imunofluorescência indireta (RIPI) mostrou $89 \%$ de positividade (título $\geq 1: 32$ ).
Tabela 1 - Idade, sexo e ocupação dos pacientes e características das lesôes, de acordo com os grupos terapêuticos.

\begin{tabular}{|c|c|c|c|}
\hline \multirow{2}{*}{$\begin{array}{l}\text { Identificação dos } \\
\text { pacientes e } \\
\text { características } \\
\text { das lesões }\end{array}$} & \multicolumn{3}{|c|}{ Grupos terapêticos } \\
\hline & pentamidina & aminosidine & glucantine \\
\hline $\begin{array}{l}\text { Pacientes (\%) } \\
\text { idade (anos) }\end{array}$ & $15(32.6)$ & $15(32,6)$ & $16(35,0)$ \\
\hline média (DP) & $23,1(8,3)$ & $29,8(13,2)$ & $24,8(12,5)$ \\
\hline intervalo & $12-40$ & $13-56$ & $13-52$ \\
\hline sexo: $M j^{\prime} F$ & $12 / 3$ & $9 / 6$ & $9 / 7$ \\
\hline \multicolumn{4}{|l|}{ ocupaçāo (\%) } \\
\hline lavrador & $12(80)$ & $13(86,7)$ & $8(50)$ \\
\hline outros & $3(20)$ & $2(13,3)$ & $8(50)$ \\
\hline \multicolumn{4}{|l|}{ durição (dias) } \\
\hline média (DP) & $40,5(25.4)$ & $35,1(22,1)$ & $63,8(23,5)$ \\
\hline interalo & $15-90$ & $8-90$ & $30-90$ \\
\hline \multicolumn{4}{|l|}{ número (\%) } \\
\hline 1 & $12(80)$ & $10(66,7)$ & $14(87,5)$ \\
\hline 2 & $3(20)$ & $5(33,3)$ & $2(12,5)$ \\
\hline \multicolumn{4}{|l|}{ local } \\
\hline MAII & $12(80)$ & $1(73,3)$ & $10(62,5)$ \\
\hline outros & $3(20)$ & $4(26,7)$ & $6(37.5)$ \\
\hline
\end{tabular}

DP $=$ desvio padrão: MMII $=$ membros inferiores

Intervalo: variação da faixa etária.

Tabela 2 - Resultados dos exames complenentares especificos realizados pré-tratanento, nos três grupos terapêticos.

\begin{tabular}{|c|c|c|c|c|c|c|}
\hline \multirow{3}{*}{$\begin{array}{l}\text { Procedimentos } \\
\text { diagnósticos }\end{array}$} & \multicolumn{6}{|c|}{ Grupos terapêuticos } \\
\hline & \multicolumn{2}{|c|}{ pentamidina } & \multicolumn{2}{|c|}{ aminosidine } & \multicolumn{2}{|c|}{ glucantine } \\
\hline & $n^{2}$ & $\%$ & $\mathrm{In}$ & $\%$ & $n^{2}$ & $\%$ \\
\hline \multicolumn{7}{|l|}{$\overline{\text { IDRM }}$} \\
\hline+ & 11 & 73,3 & 11 & 73,3 & 16 & 100 \\
\hline - & 4 & 26,7 & 4 & 26,7 & 0 & 0 \\
\hline Histopatol* & 12 & 80,0 & 10 & 66.7 & 14 & 87,5 \\
\hline \multicolumn{7}{|l|}{ Cultura } \\
\hline+ & 5 & 33,3 & 3 & 20,0 & 1 & 6,3 \\
\hline- & 4 & 26,7 & 5 & 33,3 & 3 & 18,7 \\
\hline c & 6 & 40,0 & 7 & 46,7 & 12 & 75,0 \\
\hline \multicolumn{7}{|l|}{ RIFI } \\
\hline+ & 12 & 80,0 & 14 & 93,3 & 15 & 93,7 \\
\hline - & 3 & 20,0 & 1 & 6.7 & 1 & 6,7 \\
\hline \multicolumn{7}{|l|}{ "Imprint" } \\
\hline+ & 8 & 53.3 & 8 & 53,3 & 10 & 62,5 \\
\hline- & 7 & 46.7 & 7 & 46,7 & 6 & 37,5 \\
\hline
\end{tabular}

TDRM = intradermorreação de Montenegro; $c=$ contaminadas.

* amastigotas presentes; "imprint" = impressão em lâmina.

Nas culturas dos aspirados das lesões realizadas no pós-tratamento, 84\% foram negativas e apenas $15 \%$ positivas, assim distribuídas: três culturas nos grupos 1 e 2 e apenas uma no grupo 3.

A caracterização dos isolados, utilizando-se anticorpos monoclonais para $L(V) b$ em dez fragmentos de tecidos, evidenciou a Leishmania (Viannia) braziliensis como única espécie envolvida. 
Correia D, Macêdo VO, Canalbo EM, Barral A, Magalhães AV, Abreu MVA, Orge MLGO, Marsden P. Estudo comparativo entre antimoniato de meglumina, isotianato de pentamidina e sulfato de aminosidine, no tratamento de lesões cutâneas causadas por Leishmania (Viannia) braziliensis Revista da Sociedade Brasileira de Medicina Tropical 29:447-453, set-out, 1996.

A Figura 1 mostra a dinâmica da cicatrização nos três grupos (tempo decorrido entre o início da tarapêutica até a completa cicatrização das lesões). Não houve diferença estatisticamente significante $(p=0,34)$ na dinâmica de cicatrização nos três grupos terapêuticos, através da análise de variância. Quando foram cruzados dados como dinâmica de cicatrização e localização das lesōes, houve diferença estatística significante $(p=0,03)$, pela análise de variância com o teste Student-Newman-Keuls (SNK), com alfa igual a 0,05, mostrando que as lesões de membros superiores e com duração menor que 60 dias cicatrizam mais rapidamente, independente dos grupos terapêuticos.

Nào se detectou efeitos colaterais importantes que justificassem a suspensão dos esquemas (Figura 2). As alterações bioquímicas evidenciadas pós-tratamento situaram-se dentro da faixa de variação da normalidade esperada para o método utilizado.

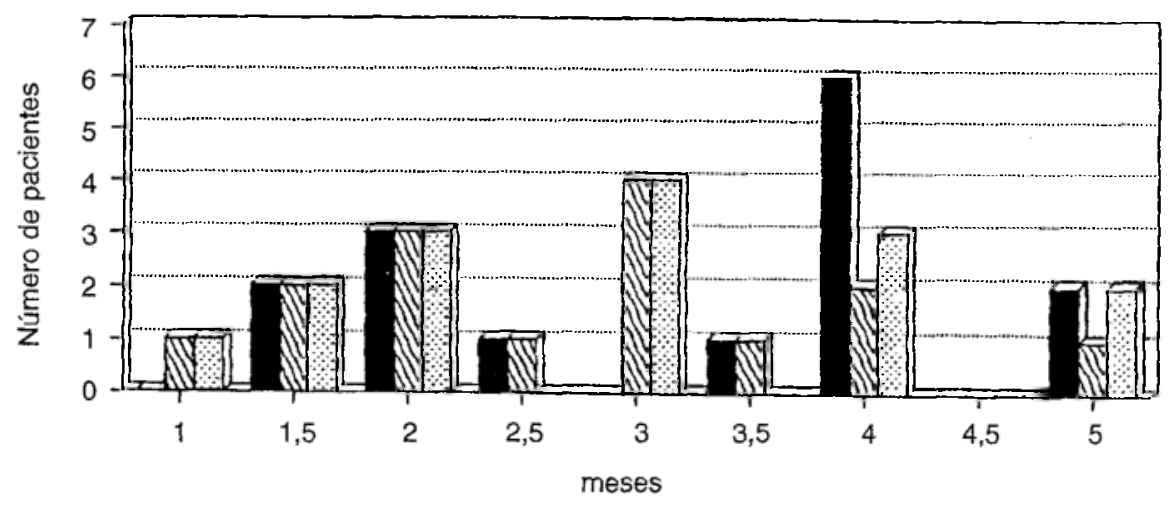

Pentamidina MIIV Aminosidine Meglumina

Figura 1 - Cicatrização das lesöes da leishmaniose tegumentar abós tratanento com pentamidina, aminosidine ou meglumina, en relação ao tempo.

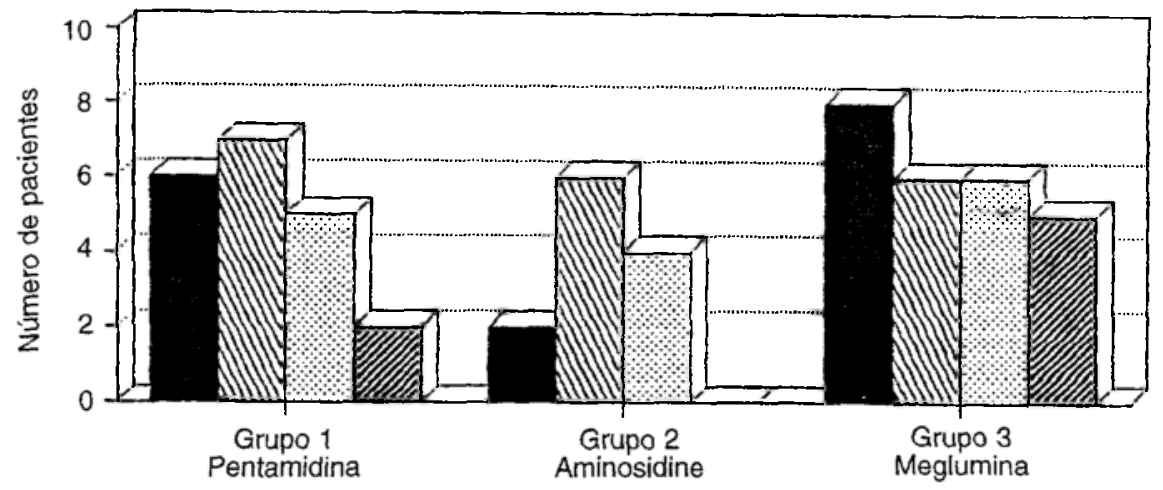

Mialgia MIII Anorexia Astenia

Figura 2 - Manifestaçōes de intolerância e efeitos colaterais em leishmanióticos tratados com os três medicamentos. 
Correia D, Macêdo VO, Carnalbo EM, Barral A, Magalbães AV, Abreu MVA. Orge MLGO, Marsden P. Estudo comparativo entre antimoniato de meglumina, isotianato de pentamidina e sulfato de aminosidine, no tratamento de lesões cutâneas causadas por Leishmania (Viannia) braziliensis. Revista da Sociedade Brasileira de Medicina Tropical 29:447-453, set-out, 1996

Após um ano de seguimento, houve cinco casos de falha terapêutica (persistência de úlceras abertas após quatro meses de tratamento), assim distribuidos: dois pacientes nos Grupos 1 e 3 e um paciente no Grupo 2.

Após três anos de seguimento, 15 pacientes foram reavaliados clinicamente, cinco do Grupo 2, todos com lesões cicatrizadas, cinco do Grupo 1, todos com lesões cicatrizadas e cinco do Grupo 3, sendo um com falha terapêutica.

\section{DISCUSSÃO}

$\mathrm{Na}$ análise dos dados encontrados na fase pré-tratamento chama a atenção a alta positividade da impressão de lâmina $(56 \%)$, quando comparada com a mencionada por outros autores ${ }^{20}$, acreditamos que se deve ao fato de $60 \%$ dos pacientes terem um período de duração de lesão menor ou igual à 3 meses.

Atualmente, para o tratamento de infecções produzidas por protozoários do gênero Leishmania, não se dispõe de compostos farmacológicos que possuam eficácia terapêutica superior à dos antimoniais pentavalentes. Estes têm o inconveniente da administração parenteral e dos efeitos colaterais. Com o intuito de se buscar alternativas terapêuticas, vários compostos têm sido utilizados, com eficácia terapêutica relativa.

O objetivo deste estudo foi o de avaliar a eficácia terapêutica de dois compostos farmacológicos ativos contra leishmânias, comparando-a com a do antimonial pentavalente.

A escolha do isotianato de pentamidina, baseou-se na sua já comprovada eficácia terapêutica ${ }^{22}$, apesar de poucos relatos na literatura ${ }^{30}{ }^{32}$. O percentual de cura por nós observado foi superior a $90 \%$ após o primeiro ano de seguimento e nos cinco pacientes reavaliados, após três anos, foi de $100 \%$. O mecanismo de ação das diamidinas aromáticas é pouco conhecido, contudo, acredita-se que cause danos irreversiveis ao complexo DNAcinetoplasto mitocondrial do parasita ${ }^{19}$ Acreditamos que a alta eficácia por nós alcançada se deva ao esquema utilizado è aos cuidados na administração.

Quanto ao sulfato de aminosidine (gabbromicina ${ }^{\circledR}$ ), um aminoglicosídio, análogo à paromomicina com atividade leishmanicida comprovada ${ }^{7725}$, acredita-se que seu mecanismo de ação seja interferir na síntese protéica ${ }^{19}$. No presente estudo, utilizamos, pela primeira vez no Brasil, a aminosidine para o tratamento de lesōes cutâneas de LTA, encorajados pelos resultados de outros autores que o fizeram para a forma visceral" ${ }^{22}$, com dose mais alta que a habitual $(20 \mathrm{mg} / \mathrm{kg} / \mathrm{dia})$ e por via intramuscular. Acreditamos ser esta explicação para a alta eficácia terapêutica por nós alcançada, superior a 90\%, após um ano de seguimento, e 100\% dos cinco pacientes reavaliados após três anos.

Quanto ao antimoniato de meglumina (glucantime ${ }^{\circledR}$ ), é vasta a literatura sobre o seu uso. Ainda não se sabe o seu exato mecanismo de ação. Acredita-se que atula inibindo a oxidação de ácidos graxos ${ }^{21}$. Neste estudo os resultados foram bons, com a dose de $10 \mathrm{mg} / \mathrm{kg} / \mathrm{dia}$, esquema proposto por MerchanHamann, 1989'16; em um ano de seguimento, alcançou-se uma eficácia terapêutica superior a 90\%, que, contudo, após três anos, caiu para 80\%. A ineficiência observada em alguns doentes tem sido atribuida à resistência do parasita ou a fatores inerentes ao hospedeiro. Contudo, há que se diferenciar fallha terapêutica de resistência, pois, ao contrário do ocorrido na forma visceral, em que é crescente o número de cepas resistentes aos antimoniais pentavalentes, na LTA a resistência não ocorre com a mesma freqüencia.

Apesar dos resultados obtidos com a pentamidina e a aminosidine serem equivalentes à aqueles obtidos com os antimoniais pentavalentes no tratamento da LTA, deve ser acentuado que há vasto respaldo na literatura para justificar que estes compostos continuem como drogas de primeira linha. A pentamidina pelo seu potencial tóxico deve continuar sendo considerada composto de segunda linha. Quanto ao aminosidine apesar dos bons resultados obtido neste ensaio a sua posição no tratamento da LTA aguarda a avaliação dos resultados e da tolerância com maior casuística e diferentes pesquisadores.

\section{SUMMARY}

With the aim of comparing the therapeutic efficacy, tolerability and toxicity of meglumine antimoniate, aminosidine sulphate and pentamidine isetbionate, a field study was conducted on randomized treatment of patients with primary cutaneous leisbmaniasis due to Leishmania (Viannia) braziliensis ( $\mathrm{L}(\mathrm{V}) \mathrm{b})$, in Conte de Pedra, $B A$, 
Correia D, Macêdo VO, Canialbo EM, Barral A, Magalhães AV, Abreu MVA, Orge MLGO, Marsden P. Estudo comparativo entre antimoniato de meglumina, isotianato de pentamidina e sulfato de aminosidine, no tratamento de lesões cutâneas causadas por Leishmania (Viannia) braziliensis. Retista da Sociedade Brasileira de Medicina Tropical 29:447-453, set-out, 1996.

from October 1992 up to January 1993. Forty six patients were treated and distributed into tbree groups, two with 15 and one with 16 subjects. All patients were submitted to clinical examination, bistopatological and immunological investigations, as diagnostic criterium. All patients were treated by intramusculary route. Group 1 receined pentamidine $4 \mathrm{mg} / \mathrm{kg} / \mathrm{etery} 2$ days, for 8 applications; Group 2 recieved aminosidine $20 \mathrm{mg} / \mathrm{kg} / \mathrm{day}$, for 20 days, and Group 3 recieteid meglumine $10 \mathrm{mg}$ Sbu/kg/day, for 20 days. Failure of therapy was defined as ulceration of the skin lesion four montbs after treatment. Such failure occurred in five cases as follows: two cases in patients of group 1 one case in patients of group 2, and two cases in group 3, after the first year of follow up. In the evaluation after three years we rewied fifteen patients, fine in each group; except for one in Group 3, all of them were cured. Statistical significance of the results beetueen the three schedules used was not verified.

Key-words: Treatment of Tegumentary Leishmaniasis. Pentazalent antimonials. Pentamidine Aminosidine.

\section{AGRADECIMENTOS}

Ao Dr. P.L. Olliaro e ao Laboratório Farmitália Carlo Erba, pela doação do sulfato de aminosidine. Ao Sr. Edinaldo Lima Lago, pelo ajuda prestada no campo.

\section{REFERÊNCIAS BIBLIOGRÁFICAS}

1. Barral A,Almeida R, Jesus AR, Netto EM, Santos IA, Jonhson WD. The relevance of characterizing leishmania from cutaneous lesions. A simple approach for isolation. Memórias do Instituto Oswaldo Cruz 82:579, 1987.

2. Barral A, Guerreiro J, Bomfim G, Correia D, BarralNetto M, Carvalho EM. Lymphadenopathy as the first sign of human cutaneous infection by Leisbmania braziliensis. American Journal of Tropical Medicine and Hygiene 53:256-259, 1995.

3. Barral-Netto M, Badaró R, Barral A, Carvalho EM. Imunologia da leishmaniose tegumentar. Revista da Sociedade Brasileira de Medicina Tropical 19:173-191, 1986.

4. Barreto AC, Cuba CC, Marsden PD, Vexenat JA, De Belder M. Padrão epidemiológico de leishmaniose americana $\mathrm{cm}$ uma região endêmica do Estado da Bahia. I. Aspectos clínicos epidemiológicos. Bulletin de la Oficina Sanitaria Panamericana 90:415, 1981.
5. Carvalho EM, Correia Filho D, Bacellar O,Almeida $\mathrm{PR}$, Lessa $\mathrm{H}$, Rocha $\mathrm{H}$. Characterization of the immune response in subjects with self-healing cutaneous leishmaniasis. American Journal of Tropical Medicine and Hygiene 53:273-277, 1995.

6. Castro C, Macêdo V,Vergara ML, Cuba C, Silveira C, Marsden PD. Leishmaniose visceral resistente respondendo ao tratamento com aminosidine. Revista da Sociedade Brasileira de Medicina Tropical 25(SuppD):85, 1992.

7. Chunge $\mathrm{CN}$, Owate J, Pamba HO, Donno L. Treatment of visceral leishmaniasis in Kenya by aminosidine alone or combined with sodium stibocluconate. Transactions of the Royal Society of Tropical Medicine and Hygiene 84:221-225, 1990.

8. Costa JML, Vale KC, França F, Saldanha ACR, Silva JO, Lago EL, Marsden PD, et al. Cura espontânea da leishmaniose cusada por Leishmania (Viannia) braziliensis em lesões cutâneas. Revista da Sociedade Brasileira de Medicina Tropical 23:205-208, 1990.

9. Cuba CCA. Leishmaniose tegumentar em área endêmica do Estado da Bahia, Brasil. Caracterização e classificação de leishmania do homem e do cão doméstico e aspectos comportamentais de Leishmania braziliensis braziliensis. Tese de Doutorado. Universidade Federal de Minas Gerais, Belo Horizonte, 1983.

10. Cuba CCA, Marsden PD, Barreto AC, Jones TC, Richards FThe use of different concentrations of leishmanial antigen in skin testing to evaluate delayed hypersensitivity in American cutaneous leishmaniasis. Revista da Sociedade Brasileira de Medicina Tropical 18:231-236, 1985.

11. Desjeux P. Human leishmaniasis: epidemiology and public health aspects. World Health Statistics Quarter 45:267-275, 1992.

12. Grimaldi Jr G, David JR, MacMahom-Pratt D. Identification of new world leishmania species characterized by serodeme analysis using monoclonal antibodies. American Journal of Tropical Medicine and Hygiene 36:270-287, 1987.

13. Magalhães AV, Moraes MAP, Raik NA, LlanosCuentas EA, Costa JML, Cuba Cuba CA, Marsden PD. Histopatologia da leishmaniose tegumentar por Leishmania braziliensis braziliensis. IV. Classificação histopatológica. Revista do Instituto de Medicina tropical de São Paulo 28:421-430, 1986. 
Correia D, Macêdo VO, Canalho EM, Barral A, Magalhães AV, Abreu MVA, Orge MLGO, Marsden P. Estudo comparativo entre antimoniato de meghmina, isotianato de pentamidina e sulfato de aminosidine, no tratamento de lesóes cutâneas causadas por Leishmania (Viannia) braziliensis. Revista da Sociedade Brasileira de Medicina Tropical 29:447-453, set-out, 1996.

14. Marsden PD. Clinical presentations of Leishmania braziliensis brazilienzis. Parasitology Today 1:129-33, 1985.

15. Marsden PD. Mucosal leishmaniasis ("espundia" Escomel, 1911). Transactions of the Royal Society of Tropical Medicine andHygiene 80:859-876, 1986.

16. Merchan-Hamann E. Ensaio terapêutico com quatro dosagens de antimonial no tratamento da leishmaniose cutânea causada por Leishmania (Viannia) braziliensis. Tese de Mestrado. Universidade de Brasília, Brasília, 1989.

17. Neal RA.The effect of antibiotics of the neomycin group on experimental cutaneous leishmaniasis. Annals of Tropical Medicine and Parasitology 54:62, 1968.

18. Netto EM, Marsden PD, Llanos-Cuentas EA, Costa JML, Cuba CC, Barreto AC. Badaró R, Johnson WD, Jones TC. Long-term follow-up of patients with Leisbmania (Viannia) braziliensis infection and treated with glucantime. Transactions of the Royal Society of Tropical Medicine and Hygiene 84:367-370, 1990.

19. Olliaro PL, Bryceson ADM. Practical progress and new drugs for changing patterns of leishmaniasis. Parasicology Today 9:323-328, 1993.

20. Organización Mundial de la Salud. Lucha contra las leishmaniasis. Série de informes técnicos 793:116-128, Genebra, OMS, 1990.

21. Ouellette M, Papadopoulou B. Mechanisms of drug resistance in leishmania. Parasitology Today 9:150-153, 1993.

22. Pradinaud R, Servans G, Sainte-Marie D, Girardeau I, Gotz W, Will F Bilan de 10 années de traitement de la leishmaniose tégumentaire par la pentamidine en Guyane Française. Nouvelles Dermatologie 10:456-611, 1991

23. Reed SG, Badaró $R$, Masur $H$, Carvalho EM, Lorenço R, Lisboa A, Teixeira R, Johnson Jr WD, Jones TC. Selection of a skin test antigen for american visceral leishmaniasis.American Journal of Tropical Medicine and Hygiene 35:79-85, 1986.
24. Revollo S, Dimier-David L, David C, Lyevre $P$, Camacho C, Dedet JP. Isoenzyme characterization of Leishmania braziliensis braziliensis isolates obtained from Bolivian and Peruvian patients. Transactions of the Royal Society of Tropical Medicine and Hygiene 86:388-391, 1992.

25. Rezzano S, Moreno G, Scorza JV. Quimioterapia experimental en hamsters por paramomicina contra dos aislados de Leisbmania mexicana y Leishmania braziliensis. Revista Cubana de Medicina Tropical 34:34-45, 1982.

26. Rosa AC, Cuba CC, Vexenat A, Barreto AC, Marsden PD. Predominance of Leishmania braziliensis braziliensis in the regions of Três Braços and Corte de Pedra, Bahia Brazil. Transactions of the Royal Society of Tropical Medicine and Hygiene 82:409-410, 1988.

27. Rossi-Bergmann B, Müller I, Godinho EB.Th1 and Th2 T-cell subsets are differentially activated by macrophages and $\mathrm{B}$ cells in murine leishmaniasis. Infection and Immunity 61:2266-2269, 1993.

28. Ryan L, Vexenat A, Marsden PD, Lainson R, Shaw JJ. The importance of rapid diagnosis of new cases of cutaneous leishmaniasis in pin-pointing the sandfly vector. Transactions of the Royal Society of Tropical Medicine and Hygiene 84:786, 1990.

29. Sampaio RN. Ensaios terapêuticos na leishmaniose tegumentar americana. Anais Brasileiros de Dermatologia 57:151-152, 1982.

30. Sampaio RN, Soares SK, Rosa AC, Netto EM, Magalhães AV, Marsden PD. Tratamento com pentamidina de seis casos da forma mucosa de leishmaniose tegumentar. Anais Brasileiros de Dermatologia 63:439-442, 1988.

31. Shaw JJ, Lainson R. A simply prepared amastigote leishmanial antigen for use in indirect fluorescent antibody test for leishmaniasis. Journal of Parasitology 63:384-385, 1977.

32. Talhari S, Sardinha JCG, Schettini APM, Arias J, Naiff RD.Tratamento da leishmaniose tegumentar americana: resultados preliminares com a pentamidina. Anais Brasileiros de Dermatologia 60:361-364. 1985. 